\title{
Dispersion Properties of Surface Polaritons in the Ferrite/Semiconductor Metamaterial in the Magnetic Field
}

\author{
I.V. Fedorin, G.S. Khripunov
}

\author{
National Technical University "Kharkiv Polytechnical Institute”, 21, Kyrpychova st., 61002 Kharkiv, Ukraine
}

(Received 04 April 2016; published online 29 November 2016)

\begin{abstract}
In the given paper, the dispersion properties of surface electromagnetic waves at a plane interface between a homogeneous medium (vacuum) and a layered periodical structure, consisting of alternating layers of ferrite and a semiconductor, placed in an external magnetic field are studied. This structure is considered in the subwavelength approximation. To describe the dielectric and magnetic properties of the material under consideration, the effective medium theory has been applied. The material parameters have been described by means of effective components of the permittivity and permeability. It was found that in a certain range of frequencies and magnitudes of external magnetic fields, in such a structure the existence of surface electromagnetic waves occur, both TM and TE polarized. Due to the peculiarities of the structure under consideration it is possible to effectively control the parameters of surface waves.
\end{abstract}

Keywords: Metamaterials, Polaritons, Ferrite, Semiconductor, Dispersion properties.

\section{INTRODUCTION}

Currently, a great interest of researchers are attracted to study the properties of surface electromagnetic waves (SEW) in the structures, that have artificially created anisotropy, in particular in metamaterials [1-4]. Properties of surface waves are largely determined by the material parameters and the state of the adjacent media, so the corresponding solutions of wave equations are widely used to study the optical properties of different materials [5-8]. By changing the design parameters of metamaterials (geometrical and physical, such as layers thicknesses, the materials forming the structure, the concentration of charge carriers in the case of materials containing free electrons, such as metals and semiconductors, etc.) one can effectively modify the dispersion characteristics of the supported waves [6].

The interest to the study of surface waves properties in metamaterials is associated with a wide range of possible practical applications of these materials in modern optoelectronic devices. The importance of these problems is due to the ability to create on the basis of plasmonic structures of novel optical devices and sensors, which have high sensitivity and compact sizes $[6,9]$.

Surface waves, i.e. waves localized near the interface, in the crystals are of two types. The first type includes dispersed surface waves at the interface with different signs of the dielectric (or magnetic) constants and frequency dispersion, called in the literature the surface polaritons (SP) (in the case of conductor surface electron plasma vibrations called surface plasmon polaritons). This is due to the imaginary transverse wave vector component. This type of surface waves occurs near the resonant frequencies [10-12]. The second type is the waves arising due to the optical anisotropy of the border materials for positive values of the dielectric (or magnetic) constants and relatively low frequency dispersion. These SEW called singular [13-18].

There are a lot of publications devoted to the study of unusual properties of surface waves in metamaterials. For example, in [6], the excitation of a well local- ized oblique surface waves on a dielectric surface with a one-dimensional array of perfectly conducting wires by the method of frustrated total internal reflection was theoretically studied. It has been found, that during the excitation of oblique waves the incident TMwave is partially polarized in the TE-wave. In [19] the features of surface plasmon-polaritons propagation at the interface of the dielectric and nanocomposite material with the metal inclusions of spherical shape were investigated.

The surface electromagnetic waves in the terahertz frequency range are of particular interest [20-22]. Terahertz range, which can be related to frequencies from one to ten $\mathrm{THz}$, is studied very little for years due to lack of sources and radiation detectors. The emergence of new sources of broadband terahertz radiation is intensified the researches of this spectral region [22]. In [23] the dispersion equation, structure of the polarization, and the energy characteristics of surface polaritons, which occur at the interface of an isotropic dielectric with an anisotropic superconductor were studied. The surface plasmon-polaritons in $\mathrm{THz}$ region of the spectrum in the case, when the period of the irregularities on the metal surface are of order of wavelength were investigated in [24].

In $[25,26]$ the possibility of surface waves existence at the boundary of the metamaterial with $\varepsilon<0$ and $\mu$ $<0$, and an isotropic medium was considered. It was found that, depending on the physical parameters of the materials at such boundaries only one surface mode with s- or a p-polarization can be excited [25, 26]. A number of works are devoted to the study of the surface wave's propagation in the photonic crystals, which is primarily due to the prospect of their application in quantum optics and optoelectronics [27-30].

In our previous studies [31-32], surface electromagnetic waves at the interface between a homogeneous medium (vacuum) and the periodic fine-stratified structure, which consist from semiconductor and dielectric layers placed in an external magnetic field, have been studied. It has been shown, that in the certain 
frequency bands and external magnetic fields, in such a structure, surface polaritons localized near the interface can exist. In [33] the properties of surface waves at the interface of a finite layered periodic structure (dielectric-semiconductor) placed in a vacuum were investigated, including the corresponding study in terms of the effective medium.

In the present study, surface electromagnetic waves (surface polaritons), which occur at the plane interface between the vacuum and biaxial metamaterial, that consist from semiconductor and ferrite layers, placed in an external magnetic field, applied parallel to the boundaries of the layers, is considered. The interest to the study of such problems is due to the fact, that the considerable practical interest represents the control of light at the nanoscale, which is a promising use of surface polaritons due to their high localization, and, as a consequence, high intensity, leading to the strengthening of a number of optical, including nonlinear effects.

\section{FERRITE $\backslash$ SEMICONDUCTOR METAMATERI- AL IN AN EXTERNAL MAGNETIC FIELD}

Let us consider a magnetoactive layered periodic structure, which consist from alternate ferrite (with thickness $d_{1}$ ) and semiconductor (with thickness $d_{2}$ ) layers. The structure is placed in an external magnetic field $H_{0}$ parallel to the boundaries of the layers (along $y$ axis). The $z$ axis runs perpendicularly to the boundaries of the layers (periodicity axis). The wave vectors lie in the $x z$ plane. In this case, we can put $\partial / \partial y=0$, omitting the dependence on the coordinate $y$ in the equations [34]. Assume that the thickness of the structure is $L$ ( $L=N d$, where $N$ is the number of periods, and $d=d_{1}+d_{2}$ is the period of the structure (Fig. 1).

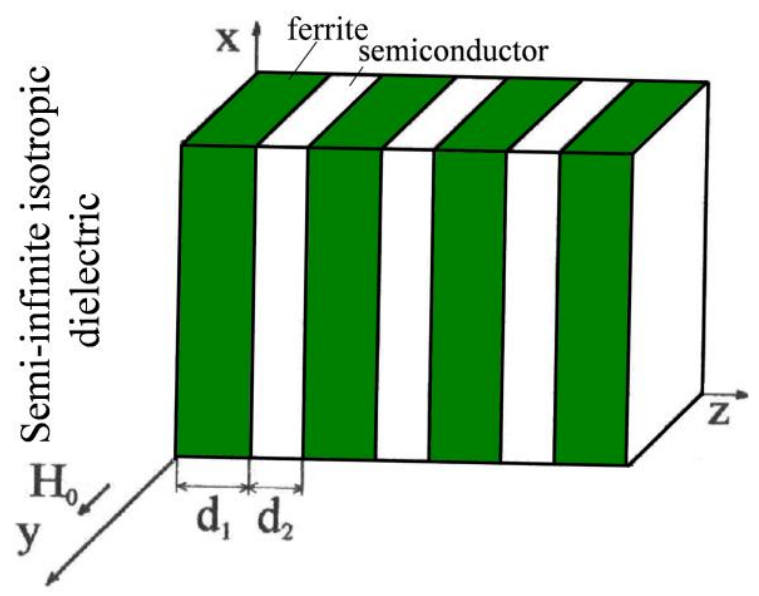

Fig. 1 - Geometry of the problem

For the chosen geometry of the structure, the Maxwell's equations can be separated into the equations for two modes with different polarizations, namely, TEpolarization with components $H_{x}, H_{z}, E_{y}$ (ordinary waves, or $H$-waves) and the TM-polarization with components $E_{x}, E_{z}, H_{y}$ (extraordinary waves, or $E$-waves). It should be noted that the external magnetic field affects the TE-wave properties in a ferrite layer only and the TM-wave properties in a semiconductor layer only.
Let us consider the case, when $\left(k_{z}^{f}\right)^{E, H} d_{1}$, $\left(k_{z}^{s}\right)^{E, H} d_{2}, k^{E, H} d<<1$ (here $\left(k_{z}^{f}\right)^{E, H},\left(k_{z}^{s}\right)^{E, H}, k^{E, H}$ are the transversal wavenumbers of the ferrite layer, semiconductor layer, and Bloch's wavenumbers of the periodic structure for $E$ - and $H$-waves, respectively. Physically it means that the period of the multilayer magnetoactive periodic structure is much less than the wavelength along $z$ axis $(d<<\lambda)$. In this case the effective medium theory can be applied, and it is possible to introduce the following effective components of permittivity and permeability [35]

$$
\begin{gathered}
\varepsilon_{x x}=\frac{\varepsilon_{F} d_{1}+\varepsilon_{f} d_{2}}{d}, \varepsilon_{y y}=\frac{\varepsilon_{F} d_{1}+\left(\varepsilon_{y y}^{s}\right) d_{2}}{d}, \\
\varepsilon_{z z}=\frac{\varepsilon_{x x} d^{2}}{d_{1} d_{2}\left(\varepsilon_{f} / \varepsilon_{F}+\varepsilon_{F} / \varepsilon_{\square}\right)+d_{1}^{2}+d_{2}^{2}}, \\
\varepsilon^{e f f}=\left(\begin{array}{ccc}
\varepsilon_{x x} & 0 & 0 \\
0 & \varepsilon_{y y} & 0 \\
0 & 0 & \varepsilon_{z z}
\end{array}\right) ; \\
\mu_{x x}=\frac{\mu_{f} d_{1}+d_{2}}{d}, \mu_{z z}=\frac{\mu_{x x} \mu_{z z}^{*}}{\mu_{x x}+\alpha \mu_{z z}^{*}}, \\
\mu_{z z}^{*}=\frac{d \mu_{f}}{\mu_{f} d_{2}+d_{1}}, \alpha=\frac{d_{1} d_{2}}{d^{2} \mu_{f}}\left(\frac{\mu_{\perp}}{\mu_{\square}}\right)^{2}, \\
\mu^{e f f}=\left(\begin{array}{ccc}
\mu_{x x} & 0 & 0 \\
0 & 1 & 0 \\
0 & 0 & \mu_{z z}
\end{array}\right) .
\end{gathered}
$$

Here $\varepsilon_{f}=\left(\left(\varepsilon_{x z}^{s}\right)^{2}+\left(\varepsilon_{x x}^{s}\right)^{2}\right) / \varepsilon_{x x}^{s}$ is the so called Voigt effective permittivity of the semiconductor layer; $\varepsilon_{F}$ is the permittivity of the ferrite layer; $\varepsilon_{x x}^{s}, \varepsilon_{y y}^{s}, \varepsilon_{x z}^{s}$ are the components of the semiconductor permittivity tensor [34]

$$
\varepsilon^{s}=\left(\begin{array}{ccc}
\varepsilon_{x x} & 0 & \varepsilon_{x z} \\
0 & \varepsilon_{y y} & 0 \\
\varepsilon_{z x} & 0 & \varepsilon_{z z}
\end{array}\right),
$$

where

$$
\begin{gathered}
\left(\varepsilon_{x x}^{s}\right)=\left(\varepsilon_{z z}^{s}\right)=\varepsilon_{\square}=\varepsilon_{0}\left(1-\frac{\omega_{P}^{2}(\omega+i v)}{\omega\left[(\omega+i v)^{2}-\omega_{C}^{2}\right]}\right), \\
\left(\varepsilon_{x z}^{s}\right)=-\left(\varepsilon_{z x}^{s}\right)=\varepsilon_{\perp}=-i \varepsilon_{0}\left(\frac{\omega_{P}^{2} \omega_{C}}{\omega\left[(\omega+i v)^{2}-\omega_{C}^{2}\right]}\right), \\
\left(\varepsilon_{y y}^{s}\right)=\varepsilon_{0}\left(1-\frac{\omega_{P}^{2}}{\omega(\omega+i v)}\right) .
\end{gathered}
$$

In the previous equations $\varepsilon 0$ is the part of the permittivity attributed to the lattice, $\omega_{p}=\sqrt{4 \pi e^{2} n_{0} / m_{\text {eff }} \varepsilon_{0}}$ 
is the plasma frequency, $\omega_{C}=e H_{0} / m_{e f f} c$ is the cyclotron frequency, $v$ is the electron collision frequency; $e$, $n_{0}$, and $m_{\text {eff }}$ are the charge, concentration, and effective mass of charge carriers. The permeability for nonmagnetic semiconductor layer is $\mu^{s}=1$.

In the ferrite layer $\mu_{F}=\mu_{\square}+\mu_{\perp}^{2} / \mu_{\square}$ is the ferrite permeability, where

$$
\begin{gathered}
\mu_{\square}=\mu_{x x}=\mu_{z z}=1+\frac{\omega_{M}\left(\omega_{H}^{2}+\omega_{r}^{2}-i \omega \omega_{r}\right)}{\omega_{H}\left(\omega_{H}^{2}+\omega_{r}^{2}-\omega^{2}-2 i \omega \omega_{r}\right)}, \\
\mu_{\perp}=\mu_{x z}=-\mu_{z x}=-\frac{i \omega \omega_{M}}{\omega_{H}^{2}+\omega_{r}^{2}-\omega^{2}-2 i \omega \omega_{r}},
\end{gathered}
$$

are the components of the ferrite permeability tensor; $\omega_{M}=2 \pi e g M / m c ; \omega_{H}=e g H_{0} / 2 m c ; g$ is the factor of spectroscopic splitting, $M$ is the saturation magnetization, $\omega_{r}$ is the relaxation frequency, $m$ is electron free mass.

It should be noted that, we applied the "resonant model" of the "saturated" ferrite to calculate the ferrite constitutive parameters, in the case, when the static magnetic field $H_{0}$ is more strong then the field of the saturation magnetization $4 \pi M$.

When static magnetic field is quite small, the "nonresonant" model of "non-saturated" ferrite should be applied. In this case the current magnetization $\mathrm{M}$ is a function of the static magnetic field [11].

Now, the Bloch wave numbers $k^{E, H}=k_{z}^{E, H}$ are the effective transverse wave numbers of a periodic structure and can be written as follows

$$
\begin{aligned}
& k_{z}^{H}=\sqrt{\left(\frac{\omega}{c}\right)^{2} \mu_{x x} \varepsilon_{y y}-\frac{\mu_{x x}}{\mu_{z z}} k_{x}^{2}}, \\
& k_{z}^{E}=\sqrt{\left(\frac{\omega}{c}\right)^{2} \varepsilon_{x x}-\frac{\varepsilon_{x x}}{\varepsilon_{z z}} k_{x}^{2}},
\end{aligned}
$$

Thus, the considered periodic structure represents the biaxial bigyrotropic metamaterial [35].

\section{SURFACE ELECTROMAGNETIC WAVES AT THE PLANE INTERFACE VACUUM- METAMATERIAL}

\subsection{Analytical Investigation}

In this section we consider surface polaritons (existence conditions), localized at the interface between semi-infinite biaxial metamaterial $(z>0)$, which is characterized by the effective permittivity and permeability (1)-(2), and a homogeneous half-space (vacuum, $z<0$ ) with . $\varepsilon_{\mathrm{v}}=\mu_{\mathrm{v}}=1$. Analytical investigation is carried out for the lossless structure, i.e. when $v=0$ and $\omega_{r}=0$.

In contrast to the general case of two semi-infinite media contact, when the damped on both sides of the interface solutions exist for only TM waves, in our case the interface vacuum-metamaterial supports both TE and TM surface
The Maxwell's equations for the metamaterial under consideration, have general form

$$
\begin{aligned}
& \operatorname{rot} \vec{E}=\frac{i \omega}{c} \mu^{\text {eff }} \vec{H}, \\
& \operatorname{rot} \vec{H}=-\frac{i \omega}{c} \varepsilon^{e f f} \vec{E} .
\end{aligned}
$$

Let us consider $E$-waves (TM) first.

Let us write the solution for the electric field vector $\mathbf{E}$ in the following form

$$
\mathbf{E}^{\mathrm{v}}=E_{0}^{\mathrm{v}} \exp \left(i k_{x} x+\chi_{\mathrm{v}} z\right), z<0 \text { (in vacuum), }
$$

$\mathbf{E}^{\mathrm{m}}=E_{0}^{\mathrm{m}} \exp \left(i k_{x} x-\chi^{E} z\right), z>0$ (in metamaterial), (6)

where $\chi_{\mathrm{v}}$ и $\chi^{E}$ are real positive values

$$
\begin{gathered}
k_{z \mathrm{v}}=i \chi_{\mathrm{v}}=i \sqrt{k_{x}^{2}-\frac{\omega^{2}}{c^{2}} \varepsilon_{\mathrm{v}}} \text { (in vacuum), } \\
k^{E}=i \chi^{E}=i \sqrt{k_{x}^{2} \frac{\varepsilon_{x x}}{\varepsilon_{z z}}-\frac{\omega^{2}}{c^{2}} \varepsilon_{x x}} \text { (in metamaterial). }
\end{gathered}
$$

Substituting the solution (5), (6) in the Maxwell equations (4), we can obtain the equations for the electric-field components $E_{x}$ and $E_{z}$. For $z<0$, i.e. in a vacuum

$$
E_{z}^{\mathrm{v}}=-i \frac{k_{x}}{\chi_{\mathrm{v}}} E_{x}^{\mathrm{v}}
$$

In the metamaterial $(z>0)$ we have

$$
E_{z}^{\mathrm{m}}=i \frac{k_{x} \varepsilon_{x x}}{\chi^{E} \varepsilon_{z z}} E_{x}^{\mathrm{m}} .
$$

Then, due to the condition of continuity of the normal component of the electric displacement at the interface $\left.D_{z 0}\right|_{z=0}=\left.D_{z 1}\right|_{z=0}\left(\left.\varepsilon_{\mathrm{v}} E_{z}^{\mathrm{v}}\right|_{z=0}=\left.\varepsilon_{z z} E_{z}^{m}\right|_{z=0}\right)$, as well as the continuity of the tangential components of the field $\mathbf{E}$, we have

$$
-\frac{\varepsilon_{\mathrm{v}}}{\chi_{\mathrm{v}}}=\frac{\varepsilon_{x x}}{\chi^{E}}
$$

Equation (11), in fact, defines the cy $\omega\left(k_{x}\right)$.Using equations (7) and (8) for the $\chi_{\mathrm{v}}$ and $\chi^{E}$, (11) can be written as

$$
\left(k_{x}^{E}\right)^{2}=\left(\frac{\omega}{c}\right)^{2}\left(\chi_{x}^{E}\right)^{2}=\left(\frac{\omega}{c}\right)^{2} \frac{\varepsilon_{z z} \varepsilon_{\mathrm{v}}\left(\varepsilon_{x x}-\varepsilon_{\mathrm{v}}\right)}{\varepsilon_{z z} \varepsilon_{x x}-\varepsilon_{\mathrm{v}}^{2}},
$$

where $\chi_{x}^{E}=\left(\left(\varepsilon_{z z} \varepsilon_{\mathrm{v}}\left(\varepsilon_{x x}-\varepsilon_{\mathrm{v}}\right)\right) /\left(\varepsilon_{z z} \varepsilon_{x x}-\varepsilon_{\mathrm{v}}^{2}\right)\right)^{1 / 2}$ is the $E$ type surface polaritons refractive index.

Equation (12) is the dispersion relation for the surface polaritons at the interface of the homogeneous half-space and the metamaterial for TM polarized waves. From (11) it also follows that the surface polaritons exist only in the region where the component $\varepsilon_{x x}$ of the metamaterial permittivity tensor is negative, i.e. $\varepsilon_{x x}<0$.

Let us note some crucial moments directly following 
from (11):

1) $k_{x} \rightarrow \infty$, when $\varepsilon_{z z} \varepsilon_{x x}-\varepsilon_{\mathrm{v}}^{2}=0$, i.e. when the following condition is satisfied $\varepsilon_{z z} \varepsilon_{x x}=\varepsilon_{\mathrm{v}}^{2}=1$;

2) $k_{x} \rightarrow 0$, where $\varepsilon_{z z} \varepsilon_{\mathrm{v}}\left(\varepsilon_{x x}-\varepsilon_{\mathrm{v}}\right)=0$, i.e. two variants are possible: $-\varepsilon_{z z}=0 ;-\varepsilon_{x x}=\varepsilon_{\mathrm{v}}=1$.

It should also be noted that when $\varepsilon_{z z}=\varepsilon_{\mathrm{v}}=1$, the dispersion equation (12) degenerates in $k_{x}^{2}=(\omega / c)^{2}$, that is, a component of the wave vector along the direction of propagation is equal to the value of the vacuum wave vector.

To sum up, it is obvious from (12) that, in the case of plane interface vacuum/biaxial metamaterial, a necessary condition of surface waves existence is negativity of $\varepsilon x x$, whereas for $\varepsilon z z$ two variants are possible. The first is the case of weak anisotropy $\left(\varepsilon_{z z}<0\right)$ when surface waves can propagate if $\varepsilon_{z z} \square \varepsilon_{x x}>\varepsilon_{\mathrm{v}}{ }^{2}$; the second is the case of strong anisotropy $\left(\varepsilon_{z z}>0\right)$ when surface waves exist at $\varepsilon_{z z}>\varepsilon_{\mathrm{v}}$ [13].

Let us similarly consider $H$-waves (TE).

Let us write the solution for the magnetic field vector $\mathbf{H}$ in the following form

$$
\begin{gathered}
\mathbf{H}^{\mathrm{v}}=H_{0}^{\mathrm{v}} \exp \left(i k_{x} x+\chi_{\mathrm{v}} z\right), z<0 \text { (in vacuum), }(13) \\
\mathbf{H}^{\mathrm{m}}=H_{0}^{\mathrm{m}} \exp \left(i k_{x} x-\chi^{H} z\right), z>0 \text { (in metamaterial), }
\end{gathered}
$$

where $\chi_{\mathrm{v}}$ и $\chi^{E}$ are real positive values

$$
k^{H}=i \chi^{H}=i \sqrt{k_{x}^{2} \frac{\mu_{x x}}{\mu_{z z}}-\frac{\omega^{2}}{c^{2}} \varepsilon_{y y} \mu_{x x}} \quad \text { (in metamateri- }
$$

al).(15)

Then the dispersion equation for TE polarized surface waves can be obtained similarly to TM waves as

$$
\left(k_{x}^{H}\right)^{2}=\left(\frac{\omega}{c}\right)^{2}\left(\chi_{x}^{H}\right)^{2}=\left(\frac{\omega}{c}\right)^{2} \frac{\mu_{z z} \mu_{\mathrm{v}}\left(\mu_{x x}-\varepsilon_{y y} \mu_{\mathrm{v}}\right)}{\mu_{z z} \mu_{x x}-\mu_{\mathrm{v}}^{2}},
$$

where $\chi_{x}^{H}=\mu_{z z} \mu_{\mathrm{v}}\left(\mu_{x x}-\varepsilon_{y y} \mu_{\mathrm{v}}\right) /\left(\mu_{z z} \mu_{x x}-\mu_{\mathrm{v}}^{2}\right)$ is the $H$-type surface polaritons refractive index.

It should be noted, that similarly to TM-waves, TE polarized surface waves exists only in the region where the magnetic permeability component $\mu_{x x}$ is negative, i.e. $\mu_{x x}<0$.

Let us note crucial moments directly following from (16):

1) $k_{x} \rightarrow \infty$, at $\mu_{z z} \mu_{x x}-\mu_{\mathrm{v}}^{2}=0$, that is, when the following condition is satisfied $\mu_{z z} \mu_{x x}=\mu_{\mathrm{v}}^{2}=1$;

2) $k_{x} \rightarrow 0$, at $\mu_{z z} \mu_{\mathrm{v}}\left(\mu_{x x}-\varepsilon_{y y} \mu_{\mathrm{v}}\right)=0$, that is, two variants are possible $\mu_{z z}=0$, and $\mu_{x x}=\varepsilon_{y y} \mu_{\mathrm{v}}$. Taking into account, that the first medium in our case is vacuum, that is $\mu_{\mathrm{v}}=\varepsilon_{\mathrm{v}}=1$, the second variant can be written as follows: $\mu_{x x}=\varepsilon_{y y}$.

As in the case of TM-polarized waves, it should also be noted for TE waves, that when $\mu_{z z}=\mu_{\mathrm{v}}=\varepsilon_{y y}=1$, the dispersion equation (16) degenerates in the $\left(k_{x}^{H}\right)^{2}=(\omega / c)^{2}$, that is, a component of the wave vector along the direction of propagation is equal to the value of the vacuum wave vector.

To sum up, a necessary condition of $H$-type surface waves existence is negativity of $\mu_{x x}$, whereas for $\mu_{z z}$ four variants are possible. The first when $\mu_{z z}<0$ and $\varepsilon_{y y}>0$, in this case surface polaritons exists if $\mu_{z z} \mu_{x x}>\mu_{\mathrm{v}}^{2}$, i.e. $\mu_{z z} \mu_{x x}>1$; the second when $\mu_{z z}<0$ and $\varepsilon_{y y}<0$, then surface polaritons exists when $\mu_{z z} \mu_{x x}>\mu_{\mathrm{v}}^{2}$ and $\operatorname{abs}\left(\mu_{x x}\right)>\varepsilon_{y y}$, or if $\mu_{z z} \mu_{x x}<\mu_{\mathrm{v}}^{2}$, i.e. $0<\mu_{z z} \mu_{x x}<1$ and $\operatorname{abs}\left(\mu_{x x}\right)<\varepsilon_{y y}$; the third occurs when $\mu_{z z}>0$ and $\varepsilon_{y y}>0$, then surface waves propagate if $\mu_{z z}>\mu_{\mathrm{v}}$, that is, when $\mu_{z z}>1$; the fourth takes place at $\mu_{z z}>0$ and $\varepsilon_{y y}<0$, when surface waves occurs if $\operatorname{abs}\left(\mu_{x x}\right)>\varepsilon_{y y}$.

Thus, the condition of surface waves existence for both polarizations depends essentially on the properties of the components of the effective permittivity and permeability tensors of the metamaterial. And, as the components of the tensors are functions of frequency, external magnetic field, thickness of layers, and physical properties of materials, that form the structure, the existence regions of surface waves are determined by the choice of values of the corresponding quantities.

In addition, it should be noted, that in this magnetic metamaterial the gyrotropy associated with the permeability characteristic tensor of the magnetic layers (ferrite layers), and electric gyrotropy is associated with the permittivity characteristic tensor of the semiconductor layers. The presence of this type of gyrotropy in the periodic structure makes it possible to effectively control with the help of an external magnetic field the band spectrum of eigenwaves of different polarizations in non-overlapping frequency bands (in the microwave range by means of magnetic gyrotropy, and in the infrared range by means of electrical gyrotropy ) [36].

Note, that the magnetic field affects the properties of the TM waves only in the semiconductor layer, and the properties of the TE waves in a layer of ferrite.

\subsection{Numerical Calculations}

In the further numerical calculations, we used the same ferrite and semiconductor parameters as in the existing theoretical and experimental papers, such as [11, 35-36]. The ferrite layer (brand 1SCH4, polycrystalline nickel ferrite $\mathrm{NiOFe} 2 \mathrm{O} 3$ ) has the following parameters $\varepsilon_{F}=11.1, g=2, d_{1}=0.05 \mathrm{~cm}$, and the saturation magnetization $M=4800 G$. As the semiconductor layer we use the indium antimonide (n-InSb) with the following ters $\varepsilon_{f}=17.8, d_{1}=0.05 \mathrm{~cm}, \omega_{p}=2 \cdot 10^{12} \mathrm{~s}^{-1}$. From the viewpoint of above analytical analysis verification, we do not take into account the electron collision 

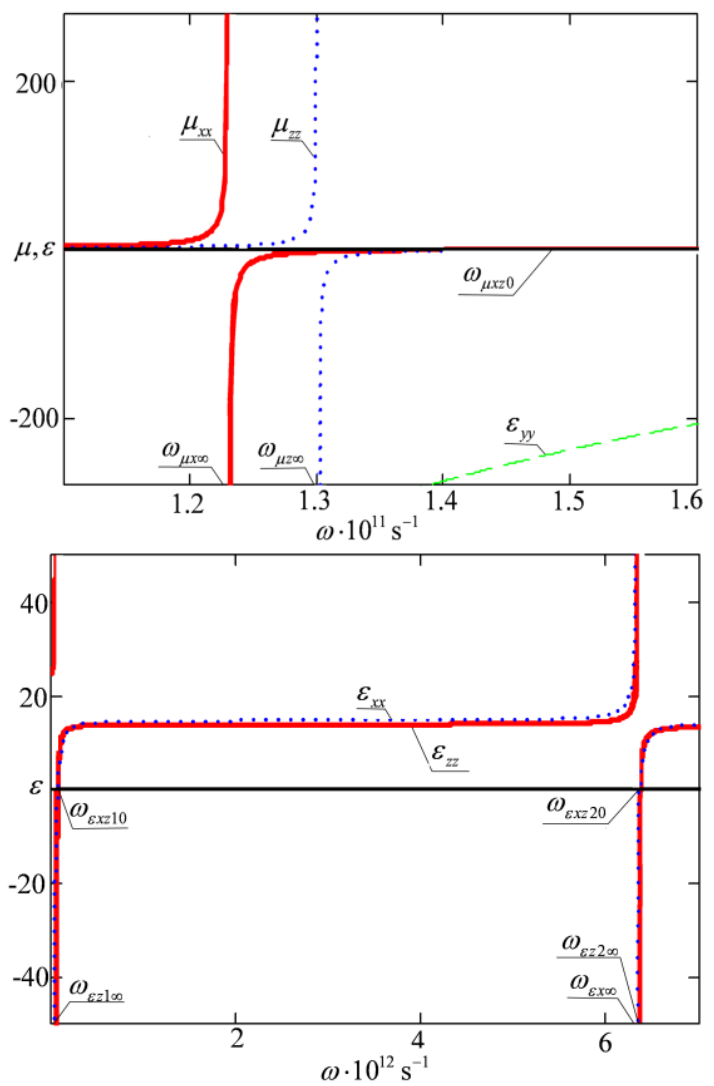

Fig. 2 - Effective permittivity and permeability of the metamaterial as a function of frequency
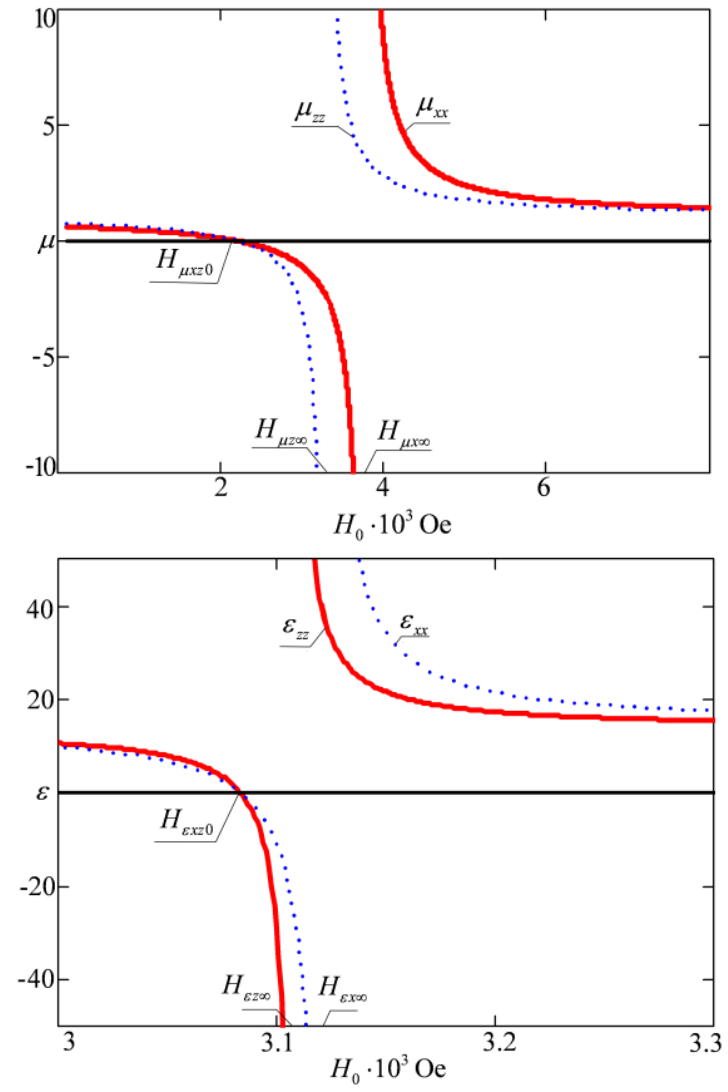

Fig. 3 - Effective permittivity and permeability of the metamaterial as a function of external magnetic field frequency in the semiconductor and magnetic damping in the ferrite layers, that is $v=0$, and $\omega_{r}=0$. Numerical analysis of dissipative processes is carried out at the end of the paper.

Let us analyze the dependence of the metamaterial effective permittivity and permeability components as a function of frequency at $H_{0}=5000$ Oe (Fig. 2), and the external magnetic field at $\omega=1 \cdot 10^{11} \mathrm{~s}^{-1}$ (when we consider the permeability components and the $\varepsilon_{y y}$ permittivity component, which are determine the properties of TE-polarized waves), and $\omega=4 \cdot 10^{12} \mathrm{~s}^{-1}$ (when we consider the $\varepsilon_{x x}$ and $\varepsilon_{z z}$ permittivity components, which are characteristic of the TM-polarized waves) (Fig. 3). Some analytical analysis of the corresponding dependencies can be found in [31, 35-36]. Here we consider the characteristic points and features that are relevant for this discussion.Let us analytically consider the effective permittivity and permeability metamaterial components, which are determine the properties of TE-waves $\left(\mu_{x x}, \mu_{z z}, \varepsilon_{y y}\right)$. Thus, at the frequency $\omega_{\mu x z 0}=\sqrt{\left(\omega_{H}+\omega_{M}\right)\left(\omega_{H}+\omega_{H} \frac{d_{f}}{d_{f}+d_{s}}\right)}$, and corresponding external magnetic field $H_{\mu x z 0}$ the effective permeability components $\mu_{x x}$ and $\mu_{z z}$ are simultaneously become zero (Fig. 2); at the ferromagnetic resonance frequency $\omega_{\mu x \infty}=\sqrt{\omega_{H}\left(\omega_{H}+\omega_{M}\right)}$, and the corresponding magnetic field the permeability component $\mu_{x x}$ and the permeability of the ferrite layer $\mu_{F}$ tend to infinity (in this case $\mu_{0} \rightarrow 0$ ).

It should be also noted, that at the frequency $\omega=\omega_{H}$ the parallel component of the ferrite permeability $\mu$ and the imaginary part of the perpendicular component $\mu_{\perp}$ tend to infinity.

At the anti-ferromagnetic resonance frequency $\omega_{a f}=\left(\omega_{H}+\omega_{M}\right)$, and at the corresponding magnetic field, the effective magnetic permeability of the ferrite layer tends to zero $\left(\mu_{F} \rightarrow 0\right)$. Finally, at the magnetic field $H_{\mu z \infty}$ and at the frequency $\omega_{\mu z \infty}$, the $\mu_{z z}$ permeability component tends to infinite. Note also, that at the considered metamaterial parameters (under the certain ferrite and semiconductor parameters), in this frequency range, the $\varepsilon_{y y}$ permittivity tensor component is negative and become zero at the frequency $\omega_{\varepsilon y 0}=\sqrt{\frac{\omega_{P}^{2} \varepsilon_{0} d_{s}}{\varepsilon_{0} d_{s}+\varepsilon_{f} d_{f}}}$. It should be noted, that arise areas in which the components of the effective permeability and permittivity are simultaneously negative, that is, it is possible to realize the negative metamaterial for TE-polarized waves.

As for $\varepsilon_{z z}$ and $\varepsilon_{x x}$ effective permittivity components, which are characteristics of the TM-polarized waves, the similar dependencies occur. Thus, at the hybrid frequency $\omega_{\varepsilon x{ }_{0}}=\sqrt{\omega_{C}^{2}+\omega_{P}^{2}}$ and corresponding 
magnetic field $H_{\varepsilon x \infty}=\left(m_{e f f} c / e\right) \sqrt{\omega^{2}-\omega_{p}^{2}}$ the parallel component of the effective metamaterial permittivity and the Voigt effective permittivity of the semiconductor tend to infinity $\left(\varepsilon_{x x}, \varepsilon_{f}^{s} \rightarrow \infty\right)$. When $\omega=\omega_{C}$ the parallel and perpendicular components of the semiconductor permittivity tensor $\varepsilon_{\perp}$ and $\varepsilon_{\mid \perp}$ tend to infinity; at the plasma frequency $\omega=\omega_{P}$ the $y$-component of the semiconductor permittivity tends to infinity $\left(\varepsilon_{y y}^{s}\right) \rightarrow \infty$.

At

frequencies

$\omega_{\varepsilon x z 10, \varepsilon x z 20}=\sqrt{\left(a_{2} \pm \sqrt{a_{2}^{2}-4 a_{1} a_{3}}\right) / 2 \cdot a_{1}}$

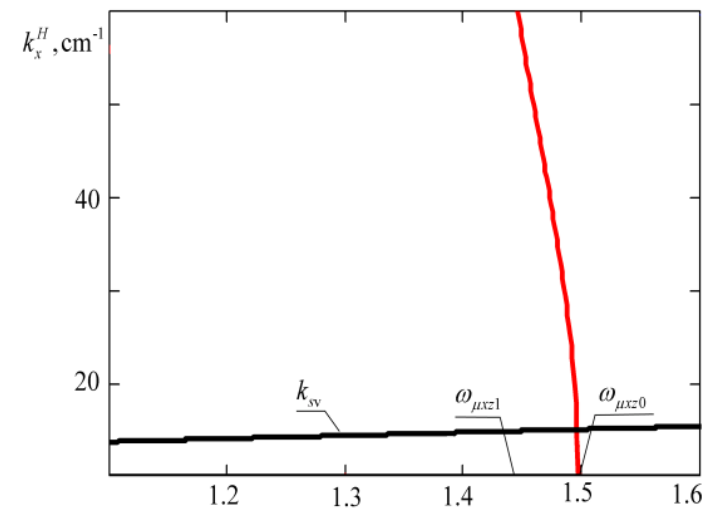

a)

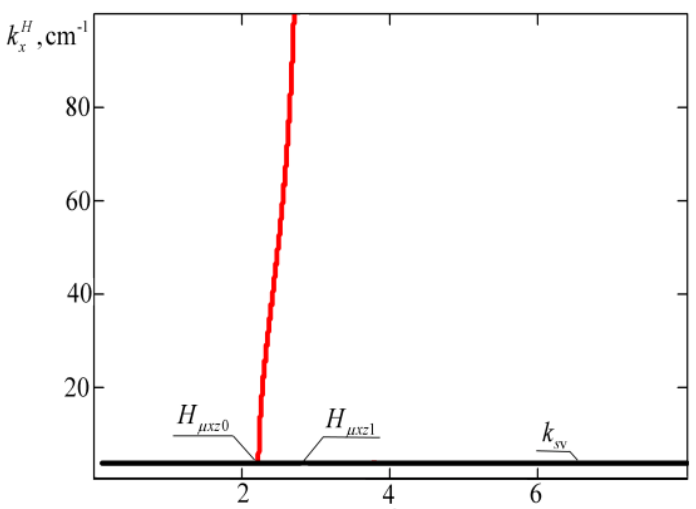

b)
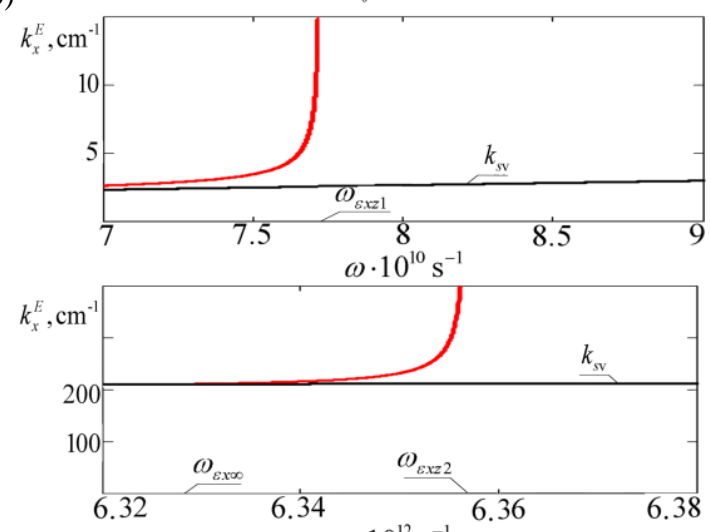

c)

$\omega \cdot 10^{12} \mathrm{~s}^{-1}$

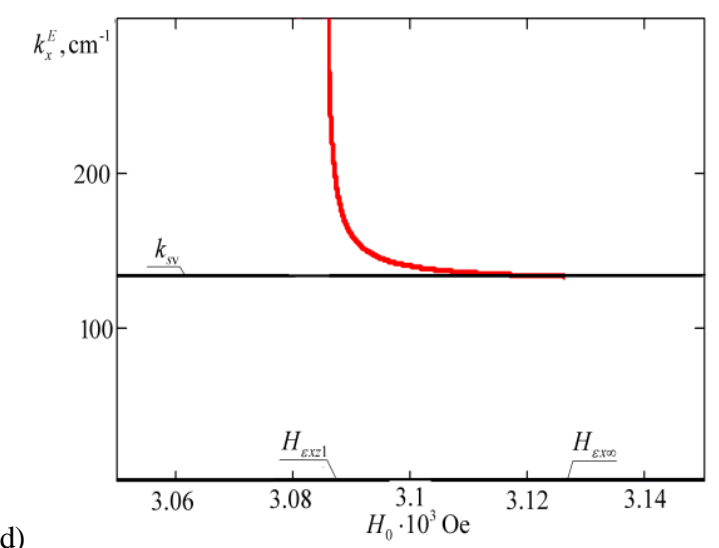

Fig. 4 - Surface waves wavenumber $k_{x}$ for TE- (a, b) and TM(c, d) polarizations as a function of: - a) and c) frequency; - b) and d) external magnetic field

where $a_{1}=d_{s} \varepsilon_{0}+d_{f} \varepsilon_{f}^{s}, \quad a_{2}=\omega_{C}^{2} a_{1}+\omega_{p}^{2}\left(d_{s} \varepsilon_{0}+a_{1}\right)$, and $a_{3}=d_{s} \varepsilon_{0} \omega_{p}^{4}$, and at the corresponding magnetic field $H_{\varepsilon x z 0}$ the components $\varepsilon_{x x}$ and $\varepsilon_{z z}$ of the metamaterial permittivity simultaneously become zero. Finally, at the magnetic field $H_{\varepsilon z \infty}$ and at the frequencies $\omega_{\varepsilon z 1 \infty, \varepsilon z 2 \infty}$ the component $\varepsilon_{z z}$ tends to infinity.

Thus, it should be noted, that a number of areas in which the condition of surface polaritons existence in the considered metamaterial (both TE and TM polarized) arises.

A necessary condition for surface polaritons existence is negativity of $\mu_{x x}$ component for TEpolarized waves and negativity of $\varepsilon_{x x}$ component for TM-polarized waves. In the presented calculations these regions are $\omega_{\mu x \infty}<\omega<\omega_{\mu x z 0} \quad$ and $H_{\mu x z 0}<H_{0}<H_{\mu x x \infty}, \quad$ where $\quad \mu_{x x}<0 ; \quad \omega<\omega_{\varepsilon x z 10}$, $\omega_{\varepsilon x \infty}<\omega<\omega_{\varepsilon x z 20}$ and $H_{\varepsilon x z 0}<H_{0}<H_{\varepsilon x \infty}$, where $\varepsilon_{x x}<0$.

Fig. 4 shows the components of the surface polaritons wave vector as a function of frequency $k_{x}(\omega)$ and the external magnetic field $k_{x}\left(H_{0}\right)$ for the TE(Fig. 4a, b) and TM- (Fig. 4c, d) polarizations, calculated from the equations (12) and (16), in the areas in which surface polaritons exist.

It should be noted, that under the selected materials parameters there are one surface polaritons dispersion curve for the TE-polarized waves, whereas two surface polaritons dispersion curves for TMpolarized waves, located above and below the plasma frequency (Fig. 4). The dispersion curves of the TMpolarized wave ( $E$-wave) tend to the light line $\left(k_{x}^{E} \rightarrow \infty\right)$ in the low frequency range, near to the frequency $\omega \rightarrow \omega_{\varepsilon x \infty}$, and magnetic field $H_{0} \rightarrow H_{\varepsilon x \infty}$ (Fig. 4c, d). On the other hand, the dispersion curves are restricted by the frequencies $\omega \rightarrow \omega_{\varepsilon x z 1, \varepsilon x z 2}$ and magnetic field $H_{0} \rightarrow H_{\varepsilon x z 1}$, at which the condition $\varepsilon_{x x} \varepsilon_{z z} \rightarrow 1$ is satisfied (these characteristic values are in the vicinity of critical frequencies $\omega \rightarrow \omega_{\varepsilon x z 10, \varepsilon x z 20}$ 
and magnetic field $H_{0} \rightarrow H_{\varepsilon x z 0}$, at which the necessary condition of TM-polarized surface polaritons existence in the system under consideration is satisfied, namely $\left.\varepsilon_{x x}=0\right)$.

The dispersion curve of the TE-polarized wave $(H$ wave) is restricted on one side by the frequency $\omega \rightarrow \omega_{\mu x z 0}$ and magnetic field $H_{0} \rightarrow H_{\mu x z 0}$, at which the necessary condition for TE-polarized surface polaritons existence in the structure under consideration is satisfied, namely $\mu_{x x}=0$; on the other hand, by the magnetic field $H_{0} \rightarrow H_{\mu x z 1}$ and the frequency $\omega \rightarrow \omega_{\mu x z 1}$, at which the condition $\mu_{x x} \mu_{z z} \rightarrow 1$ takes place.

Let us consider the properties of surface polaritons in the considered metamaterial, namely the surface polaritons phase velocity, and the penetration depth into the structure.

The surface polaritons phase velocity for both polarizations can be determined in the following way:

$$
\begin{gathered}
v_{\mathrm{ph}}^{E}=\frac{\omega}{k_{x}^{E}}=c \frac{\sqrt{\varepsilon_{z z} \varepsilon_{x x}-\varepsilon_{\mathrm{v}}^{2}}}{\sqrt{\varepsilon_{z z} \varepsilon_{\mathrm{v}}\left(\varepsilon_{x x}-\varepsilon_{\mathrm{v}}\right)}} . \\
v_{\mathrm{ph}}^{H}=\frac{\omega}{k_{x}^{H}}=c \frac{\sqrt{\mu_{z z} \mu_{x x}-\mu_{\mathrm{v}}^{2}}}{\sqrt{\mu_{z z} \mu_{\mathrm{v}}\left(\mu_{x x}-\varepsilon_{y y} \mu_{\mathrm{v}}\right)}} .
\end{gathered}
$$

Surface polaritons penetration depth (i.e. the distance at which the surface wave's field amplitude decreases $e$ times with removing from the interface) can be found as follows:

in vacuum:

$$
\delta_{\mathrm{v}}=\frac{1}{\chi_{\mathrm{v}}}
$$

in metamaterial:

$$
\delta_{\mathrm{kr}}=\frac{1}{\chi^{E, H}} .
$$

The corresponding dependencies of the surface polaritons phase velocity and the penetration depth versus frequency and external magnetic field are shown in Fig. 5 and Fig.6, respectively. Thus, the surface polaritons phase velocity for TM polarization tends to the speed of light, while the penetration depth is reduced at low frequencies, near to the frequency $\omega \rightarrow \omega_{\varepsilon x \infty}$, and magnetic field $H_{0} \rightarrow H_{\varepsilon x \infty}$ (Fig. 5 c, d; Fig. 6c, d). In general, the penetration depth for TM-polarized surface polaritons into the metamaterial in the entire region of surface polaritons existence is in the range 13000 microns (i.e. up to several structure periods). At the same time, at the other boundary of surface polaritons existence, that is near the frequency $\omega \rightarrow \omega_{\varepsilon x z 1, \varepsilon x z 2}$ and magnetic field $H_{0} \rightarrow H_{\varepsilon x z 1}$, the surface polaritons phase velocity decreases, whereas the penetration depth into the structure increases.

Similarly, the surface polaritons phase velocity of the TE polarization tends to the speed of light, while the penetration depth increases, at frequency $\omega \rightarrow \omega_{\mu x z 0}$ and magnetic field $H_{0} \rightarrow H_{\mu x z 0}$; on the other hand, at the frequency $\omega \rightarrow \omega_{\mu x z 1}$ and magnetic field $H_{0} \rightarrow H_{\mu x z 1}$, the surface polaritons phase velocity and the penetration depth into the metamaterial reduced. In general, the penetration depth for TE polarized surface polaritons also reaches several structure periods.

It should be noted, that the increase in surface waves penetration depth and retardation of the phase velocity is limited in the real structures by dissipative processes in the semiconductor and ferrite layers, which we have not taken into account at this stage. However, these effects are of great practical interest, since they may be useful in the development of various optoelectronic devices, in particular, of delay networks [8].

a)

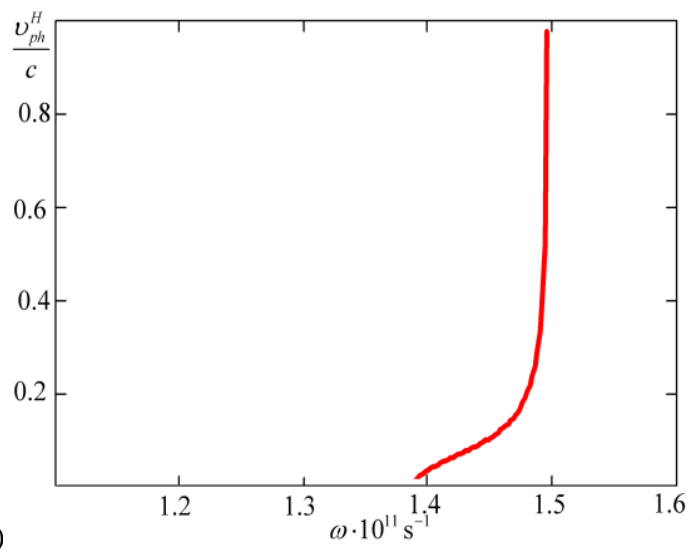

b)
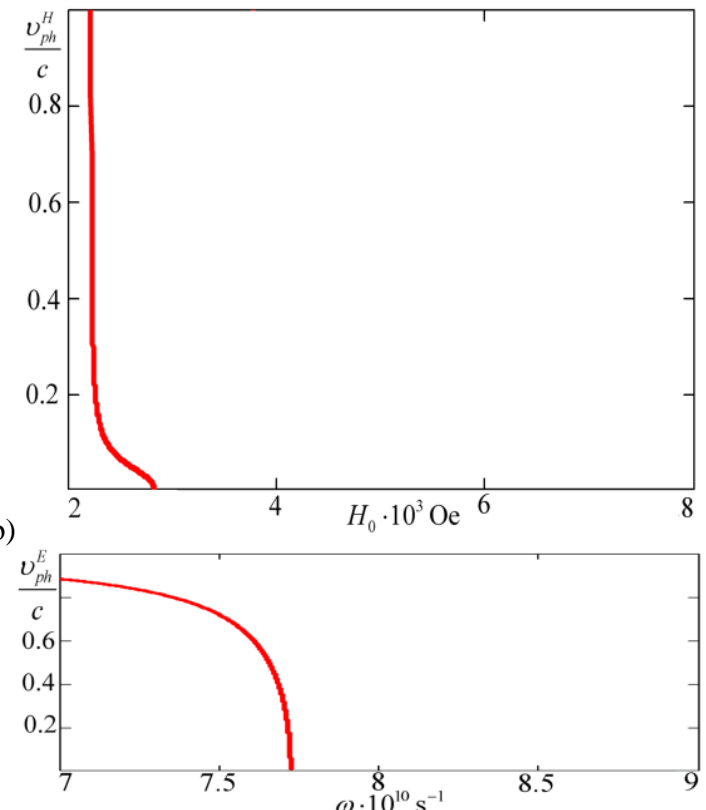

c)

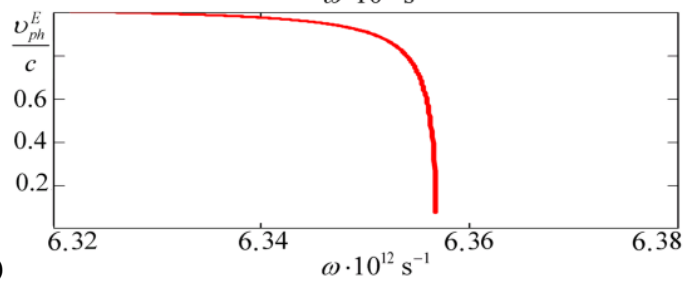




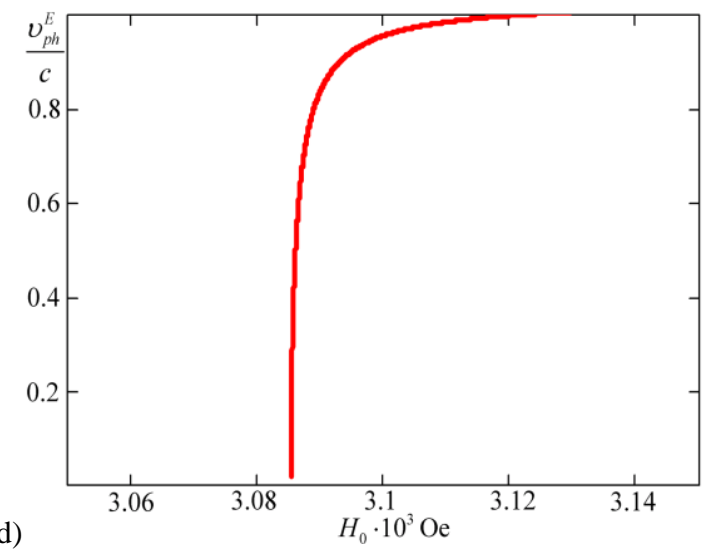

Fig. 5 - Surface waves phase velocity as a function of frequency (a, c) and external magnetic field (b, d)

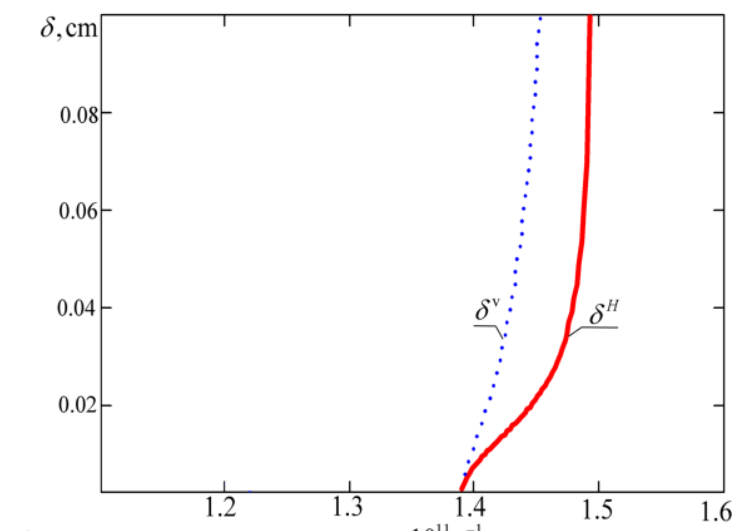

a) $\omega \cdot 10^{11} \mathrm{~s}^{-1}$

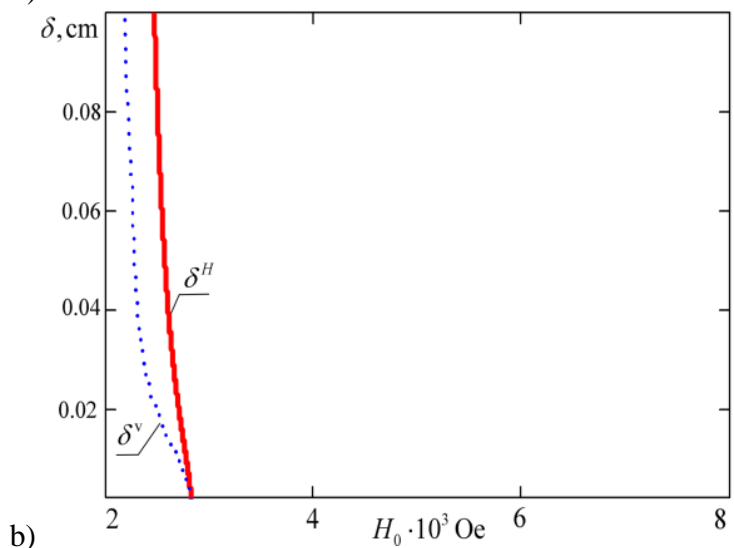

b)
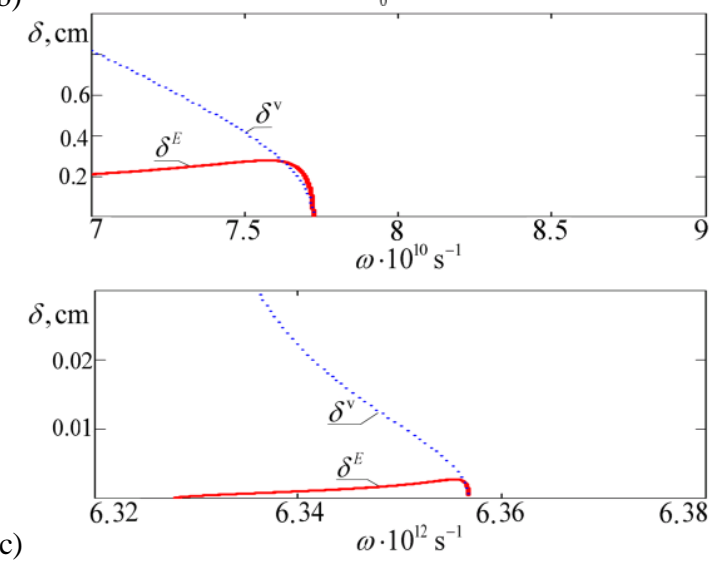

d)

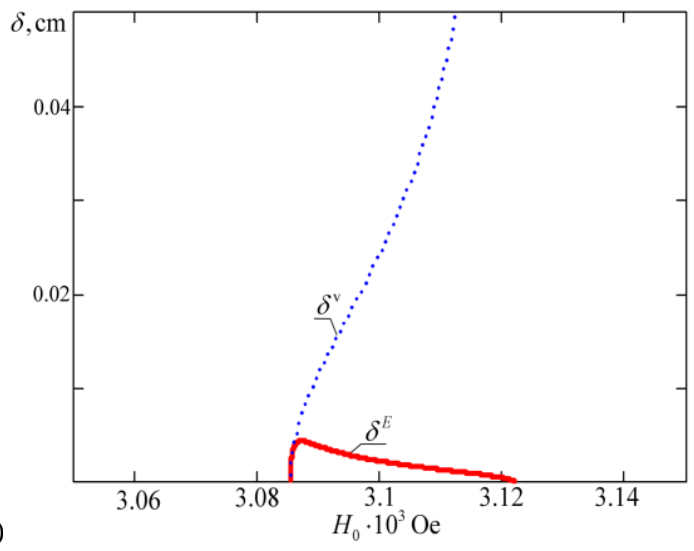

Fig. 6-Surface waves penetration depth as a function of frequency (a, c) and external magnetic field (b, d)

\subsection{The Influence of Dissipation Processes in the Ferrite and Semiconductor Layers}

Before that, we neglected magnetic damping in the ferrite layers $\left(\omega_{r}=0\right)$, and collision frequency in the semiconductor layer $(v=0)$.

Let us take now into account the dissipation in the metamaterial layers. In this case the components of the permittivity and permeability tensor of the considered metamaterial become complex quantities, and can be presented in the form $\varepsilon, \mu_{x x}=\varepsilon, \mu_{x x}^{\prime}+i \varepsilon, \mu_{x x}^{\prime \prime}$, $\varepsilon, \mu_{y y}=\varepsilon, \mu_{y y}^{\prime}+i \varepsilon, \mu_{y y}^{\prime \prime}$, and $\varepsilon, \mu_{z z}=\varepsilon, \mu_{z z}^{\prime}+i \varepsilon, \mu_{z z}^{\prime \prime}$.

Note, that in this case, the areas of surface waves existence in the system are associated with the real parts of the effective permittivity and permeability components, in particular, the necessary condition for surface waves existence is negativity of real part of $\varepsilon_{x x}$ component for E-waves $\left(\operatorname{Re} \varepsilon_{x x}<0\right)$, or negativity of $\mu_{x x}$ component for $\mathrm{H}$ waves $\left(\operatorname{Re} \mu_{x x}<0\right)$.

Fig. 7 shows the results of a numerical calculations of the real and imaginary parts of the permittivity and permeability tensor components as a function of frequency and the external magnetic field, taking into account the collision frequency in the semiconductor layer and magnetic damping in the ferrite: $v=10^{10} \mathrm{~s}^{-1}$ is the electron collisions frequency in the semiconductor; $\omega_{r}=5 \cdot 10^{8} \mathrm{c}^{-1}$ is the magnetic damping in the ferrite layer.

Note, that at the selected parameters in the considered frequency range, the $y$-component of permittivity is much less than zero $\left(\operatorname{Re} \varepsilon_{y y}<0\right)$.

As can be seen, when the dissipations are taken into account, it results in smoothing of the corresponding dependencies, absence of resonance, and, as a result, the displacement of the surface waves existence regions as TM and TE polarized, as well as a change in the surface waves field amplitude. In addition, the presence of losses in the structure leads to the restriction of the surface waves mean free path and penetration depth. However, the specific of the structure under consideration allows to effectively control the parameters of surface waves in a wide. 


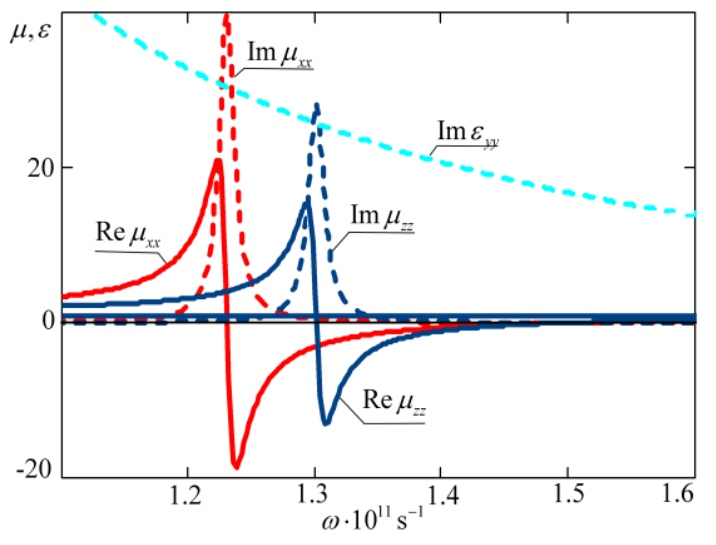

a

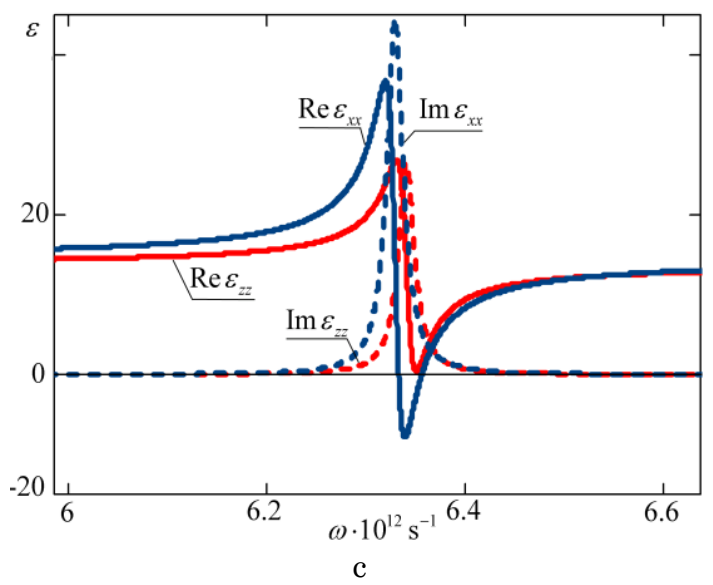

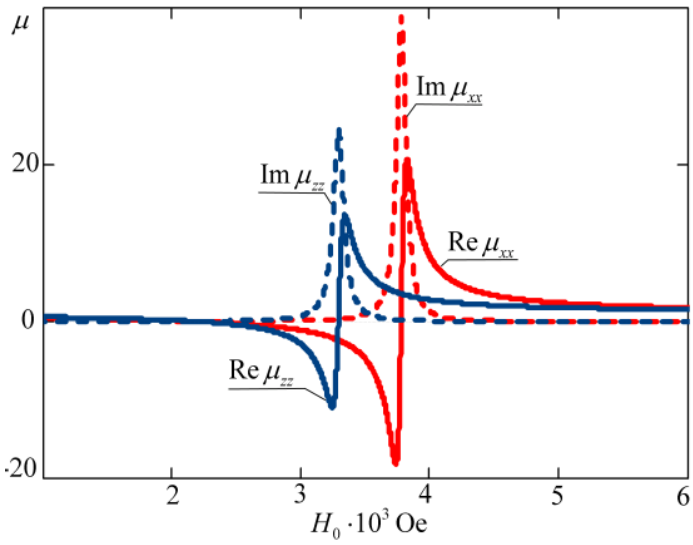

b

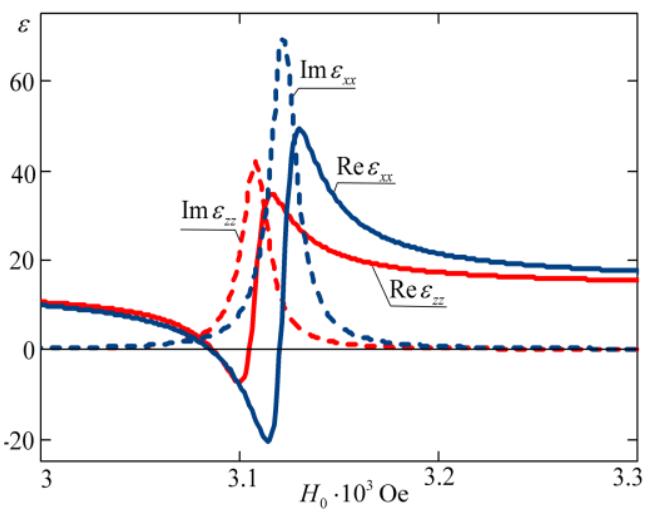

$\mathrm{d}$

Fig. 7 - Effective permittivity and permeability of the metamatiral, when the dissipations are taken into account, as a function of: - a) and c) frequency; - b) и d) external magnetic field

range of wavelengths. The nature of the changes caused by losses in the semiconductor and magnetic damping in the ferrite, require a more detailed examination, and may be one of the areas for further research

\section{CONCLUSIONS}

In the present paper, surface polaritons, which are located at the interface of a homogeneous half-space and biaxial anisotropic metamaterial in a magnetic field were studied. It has been shown, that surface polaritons can exist in a certain frequency range and magnitudes of the external magnetic field. Due to the peculiarities of the structure under consideration, where the effective components of the permittivity and permeability tensors are functions of frequency, external magnetic field, thicknesses of layers, and period of the structure, it is possible to effectively control the properties and areas of surface waves existence (in particular, to increase the surface waves penetration depth or to slowdown the phase velocity, etc.). It was found that there are two dispersion curves of surface TM po- larized polaritons and one dispersion curve of TE polarized polaritons. Areas of surface polaritons existence can be referred to areas of strong and weak anisotropy. It should be noted, that in a certain frequency bands and magnetic fields, the effective components of permittivity and permeability tensor become negative. This leads to a number of unusual and interesting from the standpoint of practical application features.

When the dissipative processes in the ferrite and in semiconductor are taking into account this leads to a smoothing of the corresponding dependencies, absence of resonances, and, as a result, to the displacement of the surface polaritons existence regions, to the change in the field amplitude. In addition, the presence of losses leads to restriction of the surface polaritons mean free path.

The results obtained in the paper are of practical interest for use in various optoelectronics devices, in the study of the surface properties, in the analysis of periodic structures, etc. On the basis of the considered plasmonic structures, metamaterials, the design and creation of new optical devices and sensors with high sensitivity and compact size is possible. 


\title{
Дисперсионные свойства поверхностных поляритонов в метаматериале феррит/полупроводник в магнитном поле
}

\author{
И.В. Федорин, Г.С. Хрипунов
}

Национальный технический университет "Харьковский политехнический институт», ул. Кирпичова, 21, 61002 Харьков, Украина

\begin{abstract}
В работе исследуются дисперсионные свойства поверхностных әлектромагнитных волн на плоской границе раздела однородной среды (вакуума) и слоисто-периодической структуры, состоящей из слоев феррита и полупроводника, помещенной во внешнее магнитное поле. Данная структура рассмотрена в субмикроволновом приближении. Для описания диэлектрических и магнитных свойств данного материала была применена теория әффективной среды. Параметры материала были описаны әффективными компонентами тензорами диэлектрической и магнитной проницаемости. Установлено, что в определенном диапазоне частот и величин внешних магнитных полей, в подобной структуре возможно распространение поверхностных әлектромагнитных волн, как ТМ, так и ТЕ поляризации. За счет специфики структуры возможно эффективное управление параметрами поверхностных волн.
\end{abstract}

Ключевые слова: Метаматериалы, Поляритоны, Феррит, Полупроводник, Дисперсионные свойства.

\section{Дисперсійні властивості поверхневих поляритонів у мета матеріалі ферит/напівпровідник у магнітному полі}

\author{
І.В. Федорін, Г.С. Хрипунов
}

\begin{abstract}
Національний технічний університет "Харківський політехнічний інститут", вул. Кирпичова, 21, 61002 Харків, Україна
\end{abstract}

\begin{abstract}
У роботі досліджуються дисперсійні властивості поверхневих електромагнітних хвиль на планарній границі розділу однорідного середовища (вакууму) і шарувато-періодичної структури, що складаеться з шарів фериту і напівпровідника, що поміщена у зовнішне магнітне поле. Дана структура розглянута в субмікрохвильовому наближенні. Для опису діелектричних і магнітних властивостей даного матеріалу була застосована теорія ефективного середовища. Параметри матеріалу були описані ефективними компонентами тензора діелектричної і магнітної проникності. Встановлено, що в певному діапазоні частот і величин зовнішніх магнітних полів в подібній структурі можливе поширення поверхневих електромагнітних хвиль, як ТМ, так і ТЕ поляризації. За рахунок специфіки структури можливе ефективне управління параметрами поверхневих хвиль.
\end{abstract}

Ключові слова: Метаматеріали, Поляритони, Ферит, Напівпровідник, Дисперсійні властивості.

\section{REFERENCES}

1. M.A. Escobar, M. Berthomé, Ch.Ma, Zh. Liu, Appl. Opt. 49, №7, A18 (2010).

2. K.L. Tsakmakidis, C. Hermann, A. Klaedtke, C. Jamois, O. Hess, Phys. Rev. B 73 No 8, 085104 (2006).

3. J.M. Pitarke, V.M. Silkin, E.V. Chulkov, P.M. Echenique, Rep. Prog. Phys 70 № 1, 1 (2007).

4. A.V. Zayats, I.I. Smolyaninov, J. Opt. A: Pure Appl. Opt. 5 No 4, S16 (2003)

5. J.B. Pendry, Phys. Rev. Let. 85 No 18, 3966 (2000).

6. Yu.O. Averkov, V.M. Yakovenko, Tech. Phys. 56 No 4, 500 (2011).

7. C.J. Zapata-Rodríguez, J.J. Miret, S. Vuković, M.R. Belić, Opt. Express 21 No 16, 19113 (2013).

8. B. Hu, Y. Zhang, Q. Wang, Nanophotonics 4, 383 (2015).

9. J.E. Toney, V.E. Stenger, S.A. Kingsley, A. Pollick, S. Sriram E. Taylor, Proc. SPIE, 8519 (2012).

10. R.F. Wallis, J.J. Brion, E. Burstein, A. Hartstein, Phys. Rev. B 9, 3424 (1974).

11. V.R. Tuz, J. Opt. 17 No 3, 035611 (2015).

12. J.J. Brion, R.F. Wallis, A. Hartstein, E. Burstein, Phys, Rev. Lett. 28, 1455 (1972).

13. V.I. Altshits, V.N. Lyubimov, JETP, 111 No 4, 591 (2010).

14. M.I. D'yakonov, Sov. Phys. JETP, 67 No 4, 714 (1988).

15. D. Artigas, L. Torner, Phys. Rev. Lett. 94, 013901 (2005).

16. W.-L. Gao, F.-Zh. Fang, Y.-M. Liu, Sh. Zhang, Light: Sci.
Appl. 4, e328 (2015).

17. Z. Jacob, E.E. Narimanov, Appl. Phys. Let. 93 No 22, 221109 (2008).

18. H.-Y. D. Yang, J. Wang, IEEE T. Antennas Propagat. 49 No 3, 444 (2001).

19. L.D. Filatov, D.G. Sannikov, D.I. Sementsov, D.A. Evseev, Phys. Solid State $\mathbf{5 6}$ No 7, 1424 (2014).

20. M. Nazarov, L.S. Mukina, A.V. Shuvaev, et al, Laser Phys. Lett. 2 No 10, 471 (2005).

21. G.N. Zhizhin, A.K. Nikitin, G.D. Bogomolov, et al, Infrared Phys. Technol. 49, 108 (2006).

22. B.A Knyazev, A.V. Kuzmin, Vestnik NGU. Series: Physics, 2 №1, 108 (2007) [in Russian].

23. S.V. Zhirnov, D.I. Sementsov, Phys. Solid State 49 No 5 , 812 (2007).

24. I.V. Yakovenko, Telecommun. Radio Eng. 51 No 6-7, 92 (1997).

25. R. Ruppin, Phys. Lett. A 277, 61 (2000).

26. I.V. Shadrivov, A.A. Sukhorukov, Yu.S. Kivshar, et al, Phys. Rev. E 69 No 1, 016617 (2004).

27. A.P. Vinogradov, A.V. Dorofeenko, S.G. Erokhin, M. Inoue, A.A. Lisyansky, A.M. Merzlikin, A.B. Granovsky, Phys. Rev. B 74 No 4, 045128 (2006).

28. J.D. Joannopoulos, R.D. Meade, J.N. Winn, Photonic Crystals: Molding the Flow of light (NJ: Princeton University 
Press, Princeton, 1995).

29. N.A. Gippius, S.G. Tikhodeev, A. Christ, J. Kuhl, H. Giessen, Phys. Solid State 47 No 1, 145 (2005).

30. A.P. Vinogradov, A.V. Dorofeenko, A.M. Merzlikhin, A.A. Lisyansky, Phys. Usp. 53, 243 (2010).

31. A.A. Bulgakov, I.V. Fedorin, Phys. Solid State 54(8), 1566 (2012).

32. I. Fedorin, V. Baibak, A. Bulgakov, Eur. Phys. J. Appl. Phys. 66 No 2, 20502 (2014).

33. V.V. Baibak, I.V. Fedorin, A.A. Bulgakov, PIER M, 32, 229 (2013).
34. F.G. Bass, A.A. Bulgakov, Kinetic and electrodynamic phenomena in classical and quantum semiconductor superlattices (Nova Science: New York: 1997).

35. A.A. Bulgakov, A.A. Girich, M.K. Khodzitsky, O.V. Shramkova, S.I. Tarapov, J.Opt. Soc. Am. B 26 No 12 , B156 (2009).

36. V.B. Kazanskiy, V.R. Tuz, V.V. Khardikov, Electrodynamic theory of composite media, (Kharkiv, Kharkiv State University, 2015) [in Russian]. 